\title{
ALMA Temporal Phase Stability and the Effectiveness of Water Vapor Radiometer
}

\author{
Satoki Matsushita ${ }^{a, b}$, Koh-Ichiro Morita ${ }^{c, b}$, Denis Barkats ${ }^{b}$, Richard E. Hills ${ }^{b}$, Ed Fomalont ${ }^{d}$, \\ and Bojan Nikolic ${ }^{e}$ \\ ${ }^{a}$ Academia Sinica Institute of Astronomy and Astrophysics, P.O. Box 23-141, Taipei 10617, \\ Taiwan, R.O.C.; \\ ${ }^{b}$ Joint ALMA Observatory, Alonso de Córdova 3107, Vitacura 763 0355, Santiago, Chile; \\ ${ }^{c}$ National Astronomical Observatory of Japan, 2-21-1 Osawa, Mitaka, Tokyo 181-8588, Japan; \\ ${ }^{d}$ National Radio Astronomy Observatory, Charlottesville, VA 22903, USA; \\ ${ }^{e}$ Astrophysics Group, Cavendish Laboratory, University of Cambridge, J J Thomson Avenue, \\ Cambridge CB3 0HE, United Kingdom
}

\begin{abstract}
Atacama Large Millimeter/submillimeter Array (ALMA) will be the world largest mm/submm interferometer, and currently the Early Science is ongoing, together with the commissioning and science verification (CSV). Here we present the temporal phase stability of the entire ALMA system; from antenna to correlator. The data, taken during the last 2 years of CSV activities, consisted of integrations on strong point sources (i.e., bright quasars) at various frequency bands, and at various baseline lengths (up to $600 \mathrm{~m}$ ). We verified the temporal phase stability of ALMA using these data. We observed a strong quasar for a long time (from a few tens of minutes, up to an hour), derived the 2-point Allan Standard Deviation after the atmospheric phase correction using the 183 $\mathrm{GHz}$ Water Vapor Radiometer (WVR) installed in each $12 \mathrm{~m}$ antenna, and confirmed that the phase stability of all the baselines reached the ALMA specification. Since we applied the WVR phase correction to all the data mentioned above, we also studied the effectiveness of the WVR phase correction at various frequencies, baseline lengths, and weather conditions. The phase stability often improves a factor of $2-3$ after the correction, and sometimes a factor of 7 improvement can be obtained. However, the corrected data still displays an increasing phase fluctuation as a function of baseline length, suggesting that the dry component (e.g., $\mathrm{N}_{2}$ and $\mathrm{O}_{2}$ ) in the atmosphere also contributes the phase fluctuation in the data, although the imperfection of the WVR phase correction cannot be discarded at this moment.
\end{abstract}

Keywords: Atacama Large Millimeter/submillimeter Array (ALMA), Commissioning and Science Verification (CSV), Phase Stability, Water Vapor Radiometer, Phase Correction

\section{INTRODUCTION}

Atacama Large Millimeter/submillimeter Array (ALMA) ${ }^{1}$ will be the world largest millimeter-/submillimeterwave interferometer, and currently the Early Science observations are ongoing, together with the Commissioning and Science Verification (CSV). One of the important tasks for CSV is to verify that the stabilities of the whole ALMA system are satisfying the specifications. Within this stability verification, verifying phase stability is one of the key missions, since the interferometric phase has the position and distribution information of the emission from target astronomical sources, and therefore critical for imaging the astronomical sources with high dynamic range and accurate positions.

The ALMA specification for the long-term temporal phase stability is defined as follows ${ }^{[2]}$ The total instrumental phase error, $\sigma$, should be below 35 femtosecond per baseline (i.e., 25 femtosecond per antenna) that is calculated with the 2-point Allan Standard Deviation (hereafter 2-p ASD),

$$
\sigma=\sqrt{\frac{<\left\{\phi_{\tau}(t+T)-\phi_{\tau}(t)\right\}^{2}>}{2}},
$$

Further author information: (Send correspondence to S.M.)

S.M.: E-mail: satoki@asiaa.sinica.edu.tw, Telephone: 886 (0)2 33652200 
Table 1. Details of the measurements. (1) Data identification number. (2) ALMA band name (number). (3) Measurement frequency in GHz. (4) Measurment date. (5) Precipitable water vapor in mm. (6) Observed source name. (7) Total observation time in minute. (8) Integration time of each data point in second. (9) Number of antennas used in the measurement.

\begin{tabular}{|c|c|c|c|c|c|c|c|c|}
\hline Data No. & Band & $\begin{array}{l}\text { Freq. } \\
{[\mathrm{GHz}]}\end{array}$ & $\begin{array}{c}\text { Date } \\
{[\mathrm{YYYY} / \mathrm{MM} / \mathrm{DD}]}\end{array}$ & $\begin{array}{l}\mathrm{PWV} \\
{[\mathrm{mm}]}\end{array}$ & Source & $\begin{array}{l}\text { Obs. Time } \\
\text { [min] }\end{array}$ & $\begin{array}{l}\text { Int. Time } \\
\text { [sec] }\end{array}$ & Ant. No. \\
\hline 1 & 3 & 87.2 & $2010 / 10 / 26$ & 0.3 & $0538-440$ & 60 & 0.1 & 7 \\
\hline 2 & & 86.3 & $2010 / 11 / 08$ & 0.5 & $0538-440$ & 60 & 0.1 & 7 \\
\hline 3 & 6 & 229.5 & $2010 / 10 / 23$ & 0.7 & $0538-440$ & 60 & 1.0 & 8 \\
\hline 4 & & & & 0.6 & $0538-440$ & 60 & 0.5 & 8 \\
\hline 5 & & & $2010 / 10 / 26$ & 0.3 & $0538-440$ & 60 & 0.1 & 7 \\
\hline 6 & & 230.6 & $2010 / 12 / 16$ & 0.6 & $0538-440$ & 60 & 1.0 & 7 \\
\hline 7 & & 229.5 & $2010 / 12 / 21$ & 2.9 & 3C279 & 60 & 1.0 & 6 \\
\hline 8 & 7 & 344.8 & $2010 / 10 / 26$ & 0.3 & $0538-440$ & 60 & 0.1 & 7 \\
\hline 9 & & 333.9 & $2011 / 10 / 06$ & 0.2 & $0522-364$ & 20 & 1.0 & 14 \\
\hline 10 & & & & 0.2 & $0522-364$ & 20 & 1.0 & 14 \\
\hline 11 & & & 2011/10/09 & 1.1 & $0538-440$ & 20 & 1.0 & 13 \\
\hline 12 & 9 & 687.7 & $2011 / 09 / 11$ & 0.2 & $1924-292$ & 20 & 1.0 & 13 \\
\hline 13 & & & $2011 / 10 / 08$ & 0.5 & $0522-364$ & 20 & 1.0 & 16 \\
\hline 14 & & & $2012 / 03 / 21$ & 0.8 & 3C279 & 20 & 1.0 & 7 \\
\hline
\end{tabular}

where $\phi_{\tau}$ is phase with a fixed averaging time, $\tau$, of 10 second and intervals, $T$, between 20 and 300 second. $t$ indicates the data sample time, and $\langle\ldots\rangle$ means the average over the data sample. This equation is almost the same as the definition of the temporal structure function for the phase fluctuation, $\frac{3}{5}$ except the temporal smoothing of 10 seconds, to measure the long-term characteristics with avoiding the short-term noise, such as high-frequency atmospheric/instrumental noise and thermal noise from various instruments.

In this proceeding, we show the ALMA system verification results of temporal phase stability. For this verification, it is crucial to eliminate all the instabilities that originate outside the ALMA system, and the dominant instability is that originates from water vapor in the atmosphere. To eliminate the instability caused by water vapor, ALMA is adopting a phase correction method using the $183 \mathrm{GHz}$ Water Vapor Radiometer (WVR) ${ }^{6}[8]$ In this proceeding, we also provide the impact of the WVR phase correction to the ALMA data.

\section{MEASUREMENTS AND DATA ANALYSIS}

To measure the long-term temporal phase stability of the whole ALMA system, we observed strong point sources, namely radio loud quasars, for $\sim 20-60$ minutes in the ALMA bands $3(\sim 86 \mathrm{GHz}), 6(\sim 230 \mathrm{GHz}), 7(\sim 345$ $\mathrm{GHz})$, and $9(\sim 690 \mathrm{GHz})$ using $6-16$ twelve meter diameter antennas under various weather conditions, which are the precipitable water vapor (PWV) of $0.2-2.9 \mathrm{~mm}$. The details of the measurements are listed in Table 1

The WVR phase correction has first been applied to the obtained data sets using the wvrgcal program. The temporal phase stability of each data set has then been calculated using a personally developed python program under the Common Astronomy Software Applications (CASA) package environment. For the 10 second data binning, if the data points are less than $70 \%$ of the number of the data points should be, this binned data point has been discarded (for example, assume the integration time of one data point is 1 second, and if the data points are less than 7 data points within 10 second, then this binned 10 second averaged data point has not been used). Phase error in femtosecond has been calculated as [rms phase in degree] / $360^{\circ} /$ [measurement frequency in Hz].

\section{EFFECTIVENESS OF THE WATER VAPOR RADIOMETER PHASE CORRECTION}

First, we present the effectiveness of the WVR phase correction to the measurement data sets in all the ALMA bands. To show this, we use spatial structure function (structure function as a function of baseline length; 

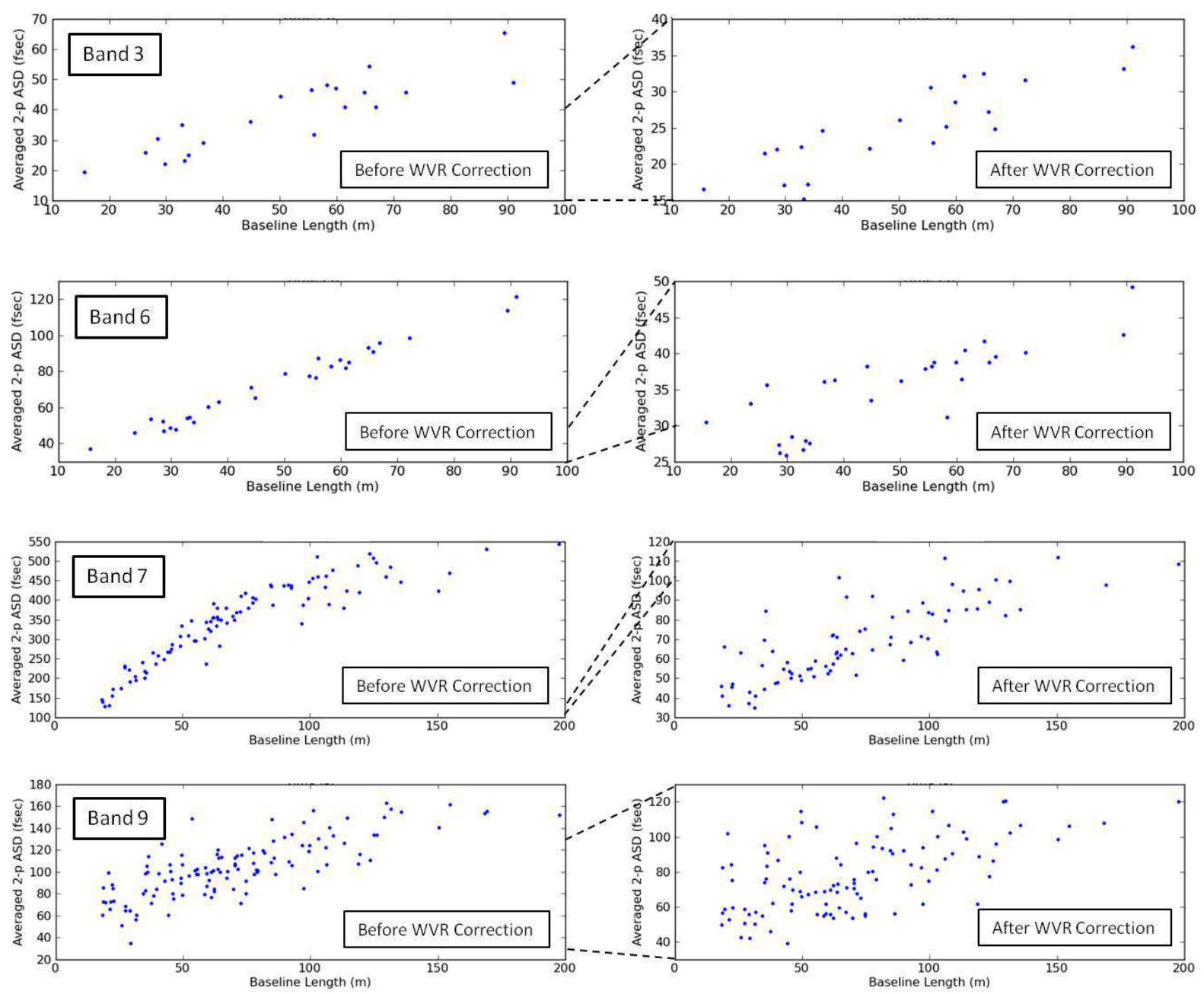

Figure 1. SSF plots before (left plots) and after (right plots) the WVR phase correction in all four ALMA bands. Here, we exhibit the results, which significantly improved the phase stability due to the WVR phase correction. Since the scaling of the vertical axes is different between the plots before and after, the same range is indicated with dashed lines. (1st row) Band 3 (data No. 2) case under PWV of $0.5 \mathrm{~mm}$. (2nd row) Band 6 (data No. 3) case under PWV of $0.7 \mathrm{~mm}$. (3rd row) Band 7 (data No. 11) case under PWV of $1.1 \mathrm{~mm}$. (4th row) Band 9 (data No. 13) case under PWV of 0.5 $\mathrm{mm}$.

hereafter SSF), which is, average the rms phase in the 2-p ASD of interval $T$ between 20 and 300 second for each baseline, and plot this as a function of baseline length. Fig. 1 displays some example SSF plots that improved significantly by the WVR phase correction. The improvement of the phase fluctuation can be seen from short $(\sim 10-20 \mathrm{~m})$ to long (up to $200 \mathrm{~m}$ ) baseline lengths, with a factor of $\sim 2$ improvement or even more; a factor of $\sim 4$ for the band 7 case in Fig. 1. The data that exhibit significant improvement are often taken under between normal to good weather conditions; the examples shown in Fig. 1 were taken under the PWV of $0.5 \sim 1.1 \mathrm{~mm}$.

The effectiveness of the WVR phase correction can also be seen in much longer baseline length of $\sim 600 \mathrm{~m}$. Fig. 2 displays the phase fluctuation plot as a function of time before and after the WVR phase correction using the data No.7 (band 6 data). The raw data (before the WVR phase correction) shows large phase fluctuation that is more than $\pm 180^{\circ}$ (Fig. 2 left plot), namely if we integrate this data set, the resultant data will lose coherence, but after the WVR phase correction, the phase fluctuation kept within $\pm 50^{\circ}$ (peak-to-peak; a factor of $\sim 4$ improvement) with a long-timescale phase drift (Fig. 2 right plot), which is good quality enough to use 

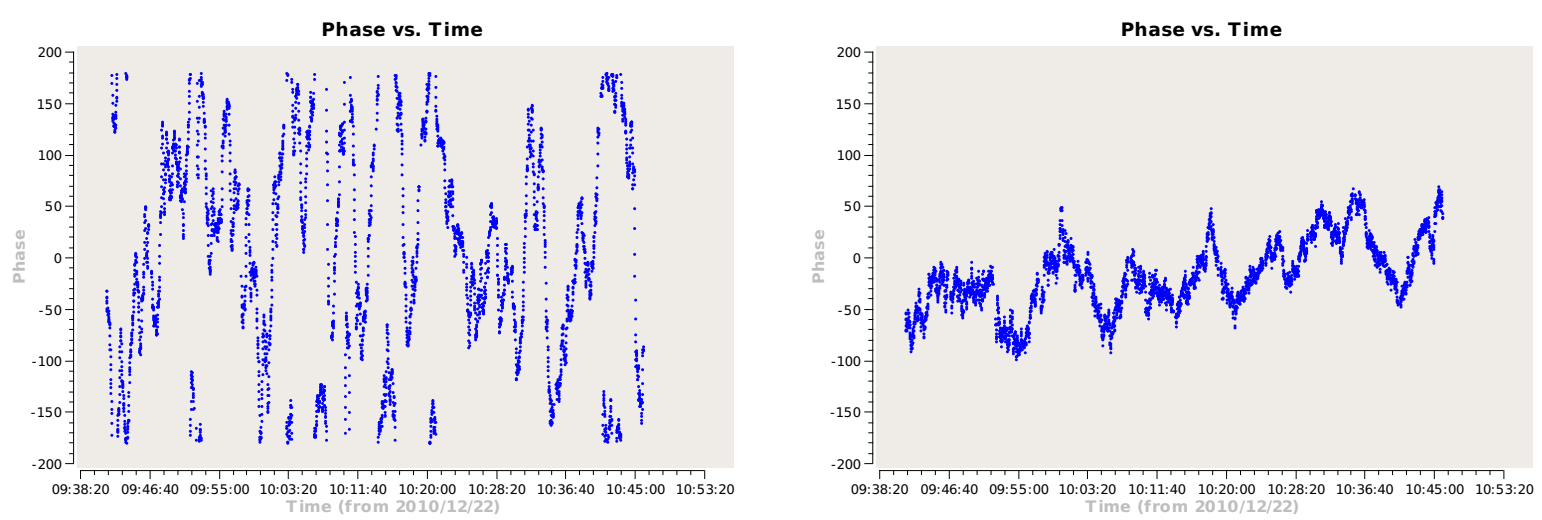

Figure 2. Time variation plots of phase before (left plot) and after (right plot) the WVR phase correction under the PWV of $2.9 \mathrm{~mm}$ and the baseline length of $\sim 600 \mathrm{~m}$. It can be clearly seen that the degree of phase fluctuation significantly improved after the WVR phase correction.

for the astronomical data analysis. It is important to mention that the weather condition was not good when this band 6 long baseline length data set has been taken (PWV $\sim 2.9 \mathrm{~mm})$, but this result prove that the data can be useful even it is taken under not really good weather condition, once the WVR phase correction has been applied.

On the other hand, some data did not improve much (Fig. 3). The data that do not show the improvement are often taken under very good weather conditions. Data No. 1, 5, 9, and 12 are the examples for this case (only the band 6 plots are shown in the 1st row of Fig. 3); these data are taken under the PWVs of $0.2 \sim 0.3$ $\mathrm{mm}$. This is understandable, since the phase fluctuation is mainly caused by water vapor in the atmosphere, and under low PWV condition, the phase fluctuation itself is already low. It is clearly seen in the maximum values of the vertical axis (SSF in femtosecond, which corresponds to the rms phase fluctuation) of Fig. 3 compared with those in the previous plots in Fig. 1. Under this low PWV condition, the difference in fluctuations of PWVs between the line of sights of two antennas (and therefore the difference in fluctuations of two WVR outputs) is too low, and therefore not much improvement has been seen by the WVR phase correction. In addition, phase fluctuation due to the dry component in the atmosphere (see the following two paragraphs below) may dominate the atmospheric phase fluctuation, and therefore resulted as less phase correction effect by WVR. But in some data set with low chance of occurrence, the phase fluctuation did not improve even under worse PWV condition of $0.6 \mathrm{~mm}$; the 2nd row of Fig. 3 displays an example of this case, probably dominated either by the dry or ice component in the atmosphere. From these examples, the WVR phase correction does not work well (or in other word, do not need to do) under very good weather conditions (PWV $\leq 0.3 \mathrm{~mm}$ ), but often works under worse conditions $(\mathrm{PWV} \sim 0.5-2.9 \mathrm{~mm}$ ). But in some rare case, it will not work even under worse conditions.

An interesting point we should note is that even under very good weather conditions or after the WVR phase correction, the rms phase fluctuation increases as a function of baseline length (Figs. 1, 3). If something caused by instruments, problems will show up as the antenna-base, not as the baseline-base, so it will not show up as a function of baseline length. For example, ALMA has the line length corrector that compensate the signal path (and therefore the phase) change of the cables for each antenna, and a problem in the line length corrector result as an antenna-base problem. This suggests that there is another component other than water vapor that cause the phase fluctuation exists in the atmosphere. The most probable component is the dry component $\left(\mathrm{N}_{2}\right.$ and/or $\mathrm{O}_{2}$ ) in the atmosphere. Phase fluctuation due to the dry component is believed to be caused by changes in density of the dry air, and the phase fluctuation on short baselines is most likely due to the changes in temperature. The temporal phase structure function of the dry component will tell the temporal density/temperature fluctuation of the dry air, and this will be an important study for the coming long baseline operation of ALMA. The existence of the dry component in the phase fluctuation means that there is a limit to correct phase fluctuation only with WVR, and some other methods are needed to correct phase fluctuation perfectly.

On the other hand, there may be other possible causes of this trend, such as the WVR phase correction is still 

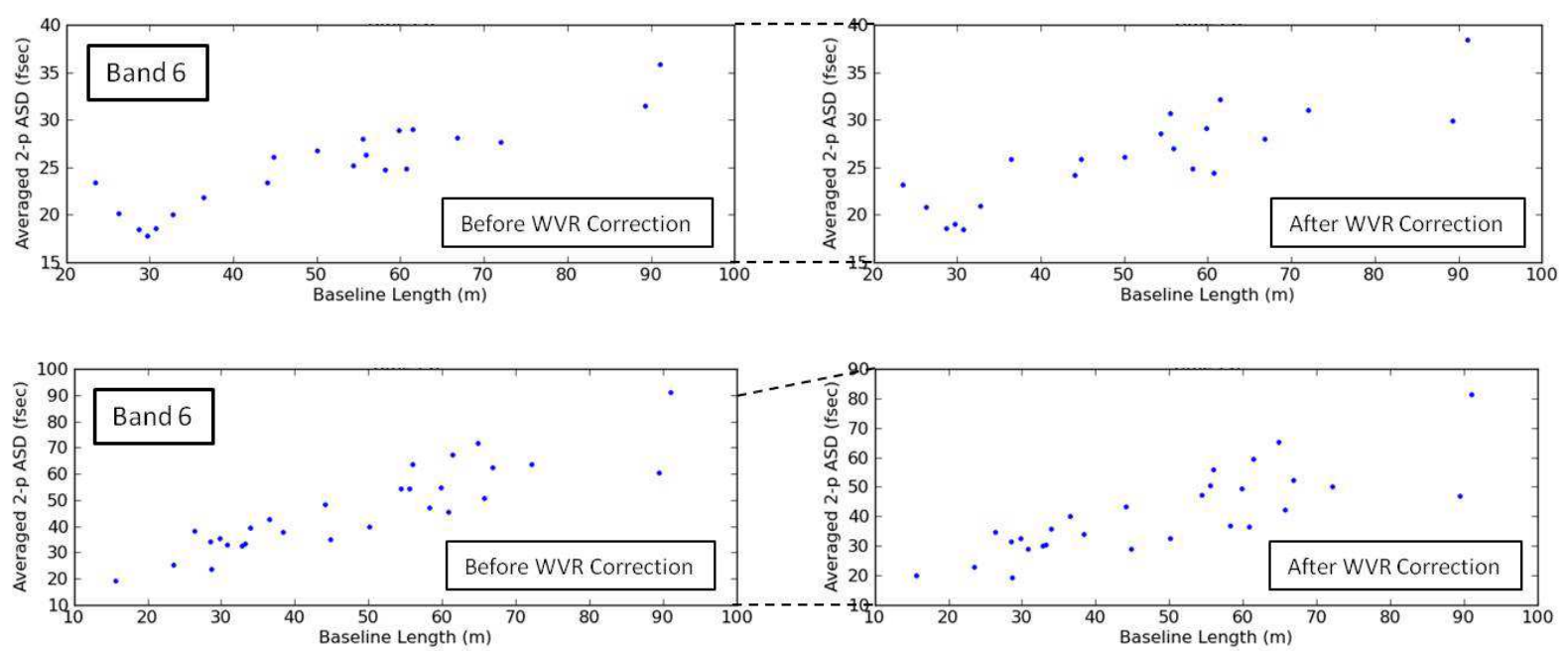

Figure 3. SSF plots before (left plots) and after (right plots) the WVR phase correction in band 6. Here, we show the results that do not improved the phase stability with the WVR phase correction. (1st row) Band 6 (data No. 5) case under PWV of $0.3 \mathrm{~mm}$. (2nd row) Band 6 (data No. 4) case under PWV of $0.6 \mathrm{~mm}$.

not optimized and therefore the residual of the phase correction appears more in longer baselines, or the WVR phase correction reached to the performance limit. However, in case of performance limit, it should show an upper limit in the temporal phase structure function at long baseline, since longer the baseline length, more difference in the atmospheric water vapor content, and eventually the difference of the water vapor contents between two antennas surpass the performance limit of the WVR phase correction. Future long baseline measurements will make this point clear.

\section{TEMPORAL PHASE STABILITY AND THE VERIFICATION OF THE ALMA SPECIFICATION}

After taking out (most of) the atmospheric effect, it is possible to verify whether the ALMA system has reached to the specification or not. Since SSF increases as a function of baseline length even after the phase correction, we should judge whether the ALMA temporal phase stability reaches the specification or not at the short baseline region.

Fig. 4 displays the 2-p ASD plots for bands 3, 6, and 7. The shaded area is the ALMA specification range. In these three bands, some or most of the baselines are within the ALMA specification range. As mentioned above, some of the baselines that are not in the ALMA specification range are long baseline length data, which we think those are affected by the dry air component, so we do not need to consider here. We therefore can conclude that the ALMA system for bands 3,6, and 7 satisfies the ALMA specification.

All the band 9 data we took, on the other hand, did not reach the ALMA specification, mainly due to low signal-to-noise $(\mathrm{S} / \mathrm{N})$ ratio of the data. In band 9, the number of bright sources with compact structure is extremely limited; all the planets are too big for band 9 even with short baseline lengths, and the minor planets are generally too weak for this purpose. Furthermore, weather conditions for band 9 are very severe, and a slight degrade of weather condition makes the data quality significantly worse. On the other hand, the current software only uses the data of $2 \mathrm{GHz}$ bandwidth and one polarization. If the software has been revised to use full bandwidth $(8 \mathrm{GHz})$ with two polarization, the sensitivity increases for $2 \sqrt{2}$, which will increase $\mathrm{S} / \mathrm{N}$, and this will be the next important update.

\section{ACKNOWLEDGMENTS}

We deeply regret the loss of our colleague, Koh-Ichiro Morita, who lost his life on May 7th, 2012, at Santiago, Chile. He significantly helped not only this work but also for various works in the ALMA project, and without 

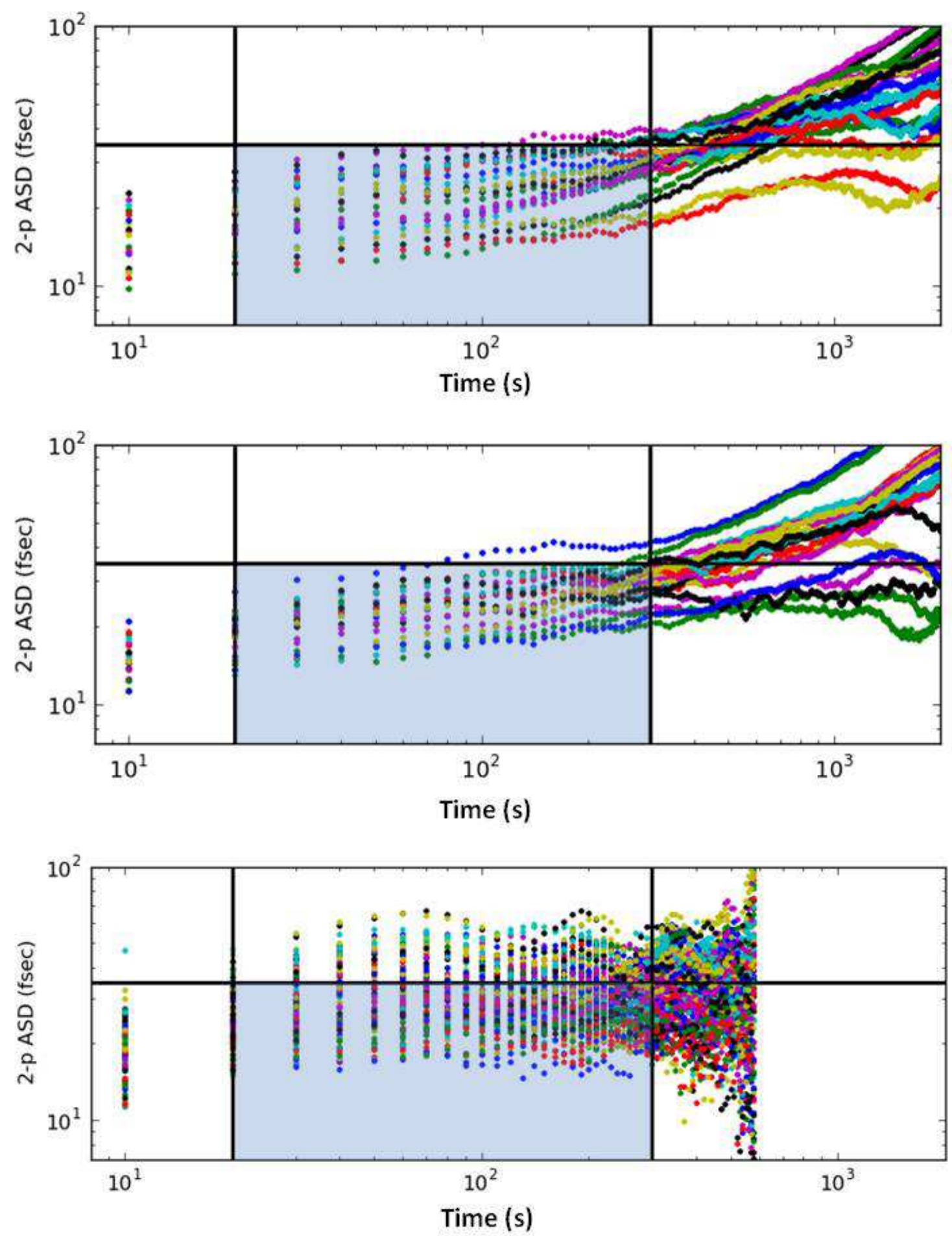

Figure 4. 2-p ASD plots that reach the specification in (1st row) band 3, (2nd row) band 6, and (3rd row) band 7. 
him, we could not achieve these results. Furthermore, he taught S.M. the basic and application of interferometry, phase correction methods, and site testings since S.M. was a graduate student at the Nobeyama Radio Observatory, and without his guidance S.M. could not be at the current position. Morita-san, we really miss you...

S.M. is supported by the National Science Council (NSC) of Taiwan, NSC 100-2112-M-001-006-MY3.

\section{REFERENCES}

[1] R. E. Hills, R. J. Kurz, and A. B. Peck, "ALMA: status report on construction and early results from commissioning," Proc. SPIE 7733, pp. 773317-773317-10, 2010.

[2] R. Sramek, and C. Haupt, "ALMA System Technical Requirements for 12m array," ALMA-80.04.00.00-005$B-S P E, 2006$

[3] V. I. Tatarskii, Wave Propagation in a Turbulent Medium, Doverm New York, 1961

[4] M. A. Holdaway, S. J. E. Radford, F. N. Owen, and S. M. Foster, "Data Processing for Site Test Interferometers," ALMA Memo bf 129, 1995

[5] A. R. Thompson, J. M. Moran, and G. W. Swenson, Jr., Interferometry and Synthesis in Radio Astronomy, Wiley-Interscience, New York, 2001 (second edition)

[6] A. Stirling, R. Hills, J. Richer, and J. Pardo, "183 GHz water vapour radiometers for ALMA: Estimation of phase errors under varying atmospheric conditions," ALMA Memo 496, 2004

[7] B. Nikolic, "Inference of Coefficients for Use in Phase Correction I," ALMA Memo 587, 2009

[8] B. Nikolic, "Inference of Coefficients for Use in Phase Correction II: Using the Observed Correlation Between Phase and Sky Brightness Fluctuations," ALMA Memo 588, 2009 